\title{
GENERATORS OF $D$-MODULES IN POSITIVE CHARACTERISTIC
}

\author{
Josep Alvarez-Montaner, Manuel Blickle, and Gennady Lyubeznik
}

\begin{abstract}
Let $R=k\left[x_{1}, \ldots, x_{d}\right]$ or $R=k\left[\left[x_{1}, \ldots, x_{d}\right]\right]$ be either a polynomial or a formal power series ring in a finite number of variables over a field $k$ of characteristic $p>0$ and let $D_{R \mid k}$ be the ring of $k$-linear differential operators of $R$. In this paper we prove that if $f$ is a non-zero element of $R$ then $R_{f}$, obtained from $R$ by inverting $f$, is generated as a $D_{R \mid k}-$ module by $\frac{1}{f}$. This is an amazing fact considering that the corresponding characteristic zero statement is very false. In fact we prove an analog of this result for a considerably wider class of rings $R$ and a considerably wider class of $D_{R \mid k}$-modules.
\end{abstract}

\section{Introduction}

Let $k$ be a field and let $R=k\left[x_{1}, \ldots, x_{d}\right]$, or $R=k\left[\left[x_{1}, \ldots, x_{d}\right]\right]$ be either a ring of polynomials or formal power series in a finite number of variables over $k$. Let $D_{R \mid k}$ be the ring of $k$-linear differential operators on $R$. For every $f \in R$, the natural action of $D_{R \mid k}$ on $R$ extends uniquely to an action on the localization $R_{f}$ via the standard quotient rule. Hence $R_{f}$ acquires a natural structure of $D_{R \mid k}$-module. It is a remarkable fact that $R_{f}$ has finite length in the category of $D_{R \mid k}$-modules. This fact has been proven in characteristic 0 by Bernstein $[2$, Cor. 1.4] in the polynomial case and by Björk [4, Thm. 2.7.12, 3.3.2] in the formal power series case. In positive characteristic the polynomial case was established by Bøgvad [8, Prop. 3.2] and the formal power series case by Lyubeznik [15, Thm. 5.9]. Consequently the ascending chain of $D_{R \mid k}$-submodules

$$
D_{R \mid k} \cdot \frac{1}{f} \subseteq D_{R \mid k} \cdot \frac{1}{f^{2}} \subseteq \cdots \subseteq D_{R \mid k} \cdot \frac{1}{f^{s}} \subseteq \cdots \subseteq R_{f}
$$

stabilizes, i.e. $R_{f}$ is generated by $\frac{1}{f^{i}}$ for some $i$. This paper is motivated by the natural question: What is the smallest $i$ such that $\frac{1}{f^{i}}$ generates $R_{f}$ as a $D_{R \mid k}-$ module?

If $k$ is a field of characteristic zero and $f \in R$ is a non-zero polynomial, the answer to this question is known: Theorem 1' of [2] shows that there exists of

Received by the editors October 24, 2004.

Research of the first author partially supported by a Fulbright grant and the Secretaría de Estado de Educación y Universidades of Spain and the European Social Funding.

The second author received funding from the DFG Schwerpunkt "Globale Methoden in der Komplexen Geometrie".

The third author gratefully acknowledges NSF support. 
a monic polynomial $b_{f}(s) \in k[s]$ and a differential operator $Q(s) \in D_{R \mid k}[s]$ such that

$$
Q(s) \cdot f^{s+1}=b_{f}(s) \cdot f^{s}
$$

for every $s$. The polynomial $b_{f}(s)$ is called the Bernstein-Sato polynomial of $f$. Let $-i$ be the negative integer root of $b_{f}(s)$ of greatest absolute value (it exists since -1 is always a root of $\left.b_{f}(s)\right)$. Then, $b_{f}(s) \neq 0$ for any integer $s<-i$, hence $f^{s} \in D_{R \mid k} \cdot f^{s+1}$ implying $\frac{1}{f^{s}} \in D_{R \mid k} \cdot \frac{1}{f^{i}}$ for all $s>i$. In particular, $R_{f}$ is $D_{R \mid k}$-generated by $\frac{1}{f^{i}}$ and, as is shown in [19, Lem. 1.3], it cannot be generated by $\frac{1}{f^{j}}$ for $j<i$. This gives a complete answer to our question in characteristic zero.

For example, consider the polynomial $f=x_{1}^{2}+\cdots+x_{2 n}^{2}$. Then, the functional equation

$$
\frac{1}{4}\left(\frac{\partial^{2}}{\partial x_{1}^{2}}+\cdots+\frac{\partial^{2}}{\partial x_{2 n}^{2}}\right) \cdot f^{s+1}=(s+1)(s+n) \cdot f^{s}
$$

shows that the Bernstein-Sato polynomial is $b_{f}(s)=(s+1)(s+n)$ [20, Cor. $3.17]$. Hence $R_{f}$ is $D_{R \mid k}$-generated by $\frac{1}{f^{n}}$ but it cannot be generated by $\frac{1}{f^{j}}$ for $j<n$.

The goal of this paper is to prove the following amazing result.

Theorem 1.1. Let $R=k\left[x_{1}, \ldots, x_{d}\right]$ or $R=k\left[\left[x_{1}, \ldots, x_{d}\right]\right]$ be either a ring of polynomials or formal power series over a field $k$ of characteristic $p>0$ and let $f \in R$ be non-zero. Then $R_{f}$ is $D_{R \mid k}$-generated by $\frac{1}{f}$.

In fact we prove this result for a considerably wider class of rings $R$ and a considerably wider class of $D_{R \mid k}$-modules.

In Section 2 we have collected for the reader's convenience some basic (and not so basic) facts about $D_{R \mid k}$-modules in characteristic $p>0$. These are needed in Section 4 and 5, whereas Section 3 can be read with minimal prior exposure to $D_{R \mid k}$-module theory.

In Section 3 we introduce a chain of ideals associated to an element $f$ of a regular $F$-finite ring $R$ of characteristic $p>0$. These ideals are of considerable interest in their own right and are likely to become useful in other contexts as well, especially in the theory of tight closure. The crucial fact is that this chain of ideals stabilizes if and only if $R_{f}$ is $D_{R \mid k}$-generated by $\frac{1}{f}$ (Corollary 3.6). This yields a very elementary proof that $R_{f}$ is $D_{R \mid k}$-generated by $\frac{1}{f}$ in the special case that $R$ is a polynomial ring over a field (Theorem 3.7 and Corollary 3.8).

In Section 4 we extend our results to all regular rings $R$ that are of finite type over an $F$-finite regular local ring of characteristic $p>0$ and to all finitely generated unit $R[F]$-modules (Theorem 4.1 and Corollary 4.4). The proof uses considerably more advanced tools than the elementary proof of Section 3 for the polynomial ring. Namely, Frobenius descent and Lyubeznik's theorem [15, Cor. 5.8] to the effect that $R_{f}$ has finite length in the category of $D_{R \mid k}$-modules are used. This result implies that the chain of ideals constructed in Section 3 stabilizes for this class of rings. These ideals squarely belong to commutative 
algebra and it is quite remarkable that the only available proof that they stabilize requires the use of $D_{R \mid k}$-modules!

In Section 5 we extend our results to the case of regular algebras $R$ of finite type over a formal power series ring $k\left[\left[x_{1}, \ldots, x_{d}\right]\right]$ where $k$ is an arbitrary field of characteristic $p>0$ (the case that $k$ is perfect is covered by the results of Section 4).

This paper combines and generalizes the results of preprints [1] and [7].

\section{Rings of differential operators and modules over them in characteristic $p>0$}

In this purely expository section we have collected some basic facts which are needed in the following sections. Throughout this section $R$ is a commutative ring containing a field of characteristic $p>0$.

2.1. Definition and elementary properties. The differential operators $\delta: R \longrightarrow R$ of order $\leq n$, where $n$ is a non-negative integer, are defined inductively as follows (cf. [10, $\S 16.8]$ ). A differential operator of order 0 is just the multiplication by an element of $R$. A differential operator of order $\leq n$ is an additive map $\delta: R \longrightarrow R$ such that for every $r \in R$, the commutator $[\delta, \tilde{r}]=\delta \circ \tilde{r}-\tilde{r} \circ \delta$ is a differential operator of order $\leq n-1$ where $\tilde{r}: R \longrightarrow R$ is the multiplication by $r$. The sum and the composition of two differential operators are differential operators, hence the differential operators form a ring which is a subring of $\operatorname{End}_{\mathbb{Z}} R$. We denote this ring $D_{R}$.

If $k \subseteq R$ is a subring, we denote by $D_{R \mid k} \subseteq D_{R}$ the subring of $D_{R}$ consisting of all those differential operators that are $k$-linear. Since every additive map

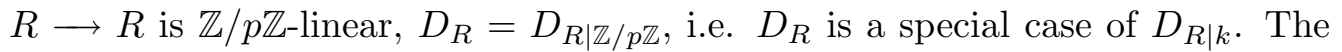
ring homomorphism $R \longrightarrow D_{R \mid k}$ that sends $r \in R$ to the multiplication by $r$ makes $R$ a subring of $D_{R \mid k}$.

By a $D_{R \mid k}$-module we always mean a left $D_{R \mid k}$-module. For example, $R$ with its natural $D_{R \mid k}$-action is a $D_{R \mid k}$-module. If $M$ is a $D_{R \mid k}$-module and $S \subset R$ is a multiplicatively closed set, then $M_{S}$ has a unique $D_{R \mid k}$-module structure such that the natural localization map $M \longrightarrow M_{S}$ is a $D_{R \mid k}$-module homomorphism [15, Ex. 5.1]. In particular, $R_{f}$ carries a natural $D_{R \mid k}$-module structure for every $f \in R$.

Every differential operator $\delta \in D_{R}$ of order $\leq p^{s}-1$ is $R^{p^{s}}$-linear, where $R^{p^{s}} \subseteq R$ is the subring consisting of all the $p^{s}$-th powers of all the elements of $R[21,1.4 .8 \mathrm{a}]$. In other words, $D_{R}$ is a subring of the ring

$$
\bigcup_{s} D_{R}^{(s)}
$$

where $D_{R}^{(s)}=\operatorname{End}_{R^{p^{s}}}(R)$. In particular, this implies that if $k$ is not just $\mathbb{Z} / p \mathbb{Z}$, but any perfect subfield of $R$, i.e. $k \subseteq R^{p^{s}}$ for every $s$, then every $\delta \in D_{R}$ is $k$-linear, i.e. $D_{R}=D_{R \mid k}$. 
Let $k\left[R^{p^{s}}\right]$ be the $k$-subalgebra of $R$ generated by the $p^{s}$-th powers of all the elements of $R$. If $R$ is a finite $k\left[R^{p}\right]$-module, then $[21,1.4 .9]$

$$
D_{R \mid k}=\bigcup_{s} \operatorname{End}_{k\left[R^{p^{s}}\right]}(R) .
$$

The ring $R$ is called $F$-finite if $R$ is a finitely generated $R^{p}$-module. Since $D_{R}=D_{R \mid \mathbb{Z} / p \mathbb{Z}}$ and $\mathbb{Z} / p \mathbb{Z}\left[R^{p^{s}}\right]=R^{p^{s}}$, it follows that if $R$ is $F$-finite, then

$$
D_{R}=\bigcup_{s} D_{R}^{(s)}
$$

If $R=k\left[x_{1}, \ldots, x_{d}\right]$ or $R=k\left[\left[x_{1}, \ldots, x_{d}\right]\right]$ is either the ring of polynomials or the ring of formal power series over $k$, then $D_{R \mid k}$ is the ring extension of $R$ generated by the differential operators $D_{t, i}=\frac{1}{t !} \frac{\partial^{t}}{\partial x_{i}^{t}}$ where $\frac{\partial^{t}}{\partial x_{i}^{t}}$ is the $t$-th $k$-linear partial differentiation with respect to $x_{i}$, i.e. $D_{t, i}\left(x_{i}^{s}\right)=0$ if $s<t$ and $D_{t, i}\left(x_{i}^{s}\right)=\left(\begin{array}{l}t \\ s\end{array}\right) x_{i}^{s-t}$ if $s \geq t[10, \S 16.11],[15$, Ex. 5.3d,e]. If $k$ is perfect, i.e. $\bigcup_{s} D_{R}^{(s)}=D_{R}=D_{R \mid k}$, then $D_{R}^{(s)}$ is the ring extension of $R$ generated by the operators $D_{t, i}$ with $t<p^{s}$.

2.2. Frobenius descent. The exposition in this subsection is based on Chapter 3.2 of [5]. We state and prove the basic result but refer to [5] for all the straightforward (but tedious) compatibilities one has to check.

Frobenius descent has been used by a number of authors; see for example S.P. Smith [18, 17], B. Haastert [11, 12] and R. Bøgvad [8]. Its precursor is Cartier descent ${ }^{1}$ as described, for example, by N. M. Katz [13, Thm. 5.1]. A big generalization has recently been given by P. Berthelot [3]

In the basic form used here Frobenius descent is based on the fact that a ring $R$ is Morita equivalent to the algebra of $n \times n$ matrices with entries in $R$. That is $R$ and $\operatorname{Mat}_{n \times n}(R)$ have equivalent module categories. Namely, $\operatorname{Mat}_{1 \times n}(R)$, the rows of length $n$ with entries from $R$ (resp. $\operatorname{Mat}_{n \times 1}(R)$, the columns of length $n$ with entries from $R$ ) is an $R$-Mat Man $_{n \times n}(R)$-bimodule (resp. a $\operatorname{Mat}_{n \times n}(R)-R$ bimodule) and the maps

$$
\begin{gathered}
\operatorname{Mat}_{1 \times n}(R) \otimes_{\operatorname{Mat}_{\mathrm{n} \times \mathrm{n}}(\mathrm{R})} \operatorname{Mat}_{n \times 1}(R) \longrightarrow R \\
\operatorname{Mat}_{n \times 1}(R) \otimes_{R} \operatorname{Mat}_{1 \times n}(R) \longrightarrow \operatorname{Mat}_{n \times n}(R)
\end{gathered}
$$

that send $A \otimes B$ to the matrix product $A B$ are isomorphisms, hence the functors

$$
\begin{gathered}
\operatorname{Mat}_{1 \times n}(R) \otimes_{\operatorname{Mat}_{n \times n}(R)}(\ldots): \operatorname{Mat}_{n \times n}(R)-\bmod \longrightarrow R-\bmod \\
\operatorname{Mat}_{n \times 1}(R) \otimes_{R}\left(\_\right): R-\bmod \longrightarrow \operatorname{Mat}_{n \times n}(R)-\bmod
\end{gathered}
$$

are inverses of each other and establish an equivalence of categories.

\footnotetext{
${ }^{1}$ It states that $F^{*}$ is an equivalence between the category of $R$-modules and the category of modules with integrable connection and $p$-curvature zero. The inverse functor of $F^{*}$ on a module with connection $(M, \nabla)$ is in this case given by taking the horizontal sections ker $\nabla$ of $M$. As an $R$-module with integrable connection and $p$-curvature zero is nothing but a $D_{R}^{(1)}$-module, Cartier descent is just the case $e=1$ of Proposition 2.1.
} 
Let $R^{(s)}$ be the abelian group of $R$ with the usual left $D_{R}^{(s)}$-module structure (and hence the usual left $R$-structure) and with the right $R$-module structure defined by $r^{\prime} r=r^{p^{s}} r^{\prime}$ for all $r \in R$ and $r^{\prime} \in R^{(s)}$. Thus $R^{(s)}$ is a $D_{R}^{(s)}-R_{-}$ bimodule. We define a structure of $R-D_{R}^{(s)}$-bimodule on $\operatorname{Hom}_{R}^{\mathrm{r}}\left(R^{(s)}, R\right)$ where Hom $^{\mathrm{r}}$ denotes the homomorphisms in the category of right $R$-modules as follows. For $\delta \in D_{R}^{(s)}, \varphi \in \operatorname{Hom}_{R}^{\mathrm{r}}\left(R^{(s)}, R\right)$ and $r \in R$ the product $r \cdot \varphi \cdot \delta$ is the composition $\tilde{r} \circ \varphi \circ \delta$ where $\delta$ acts on the left on $R^{(s)}$ and $\tilde{r}$ is the multiplication by $r$ on $R$. The identification of $D_{R}^{(s)}$ with $\operatorname{Hom}_{R}^{\mathrm{r}}\left(R^{(s)}, R^{(s)}\right)$ shows that this composition $\tilde{r} \circ \varphi \circ \delta$ is indeed in $\operatorname{Hom}_{R}^{\mathrm{r}}\left(R^{(s)}, R\right)$. Thus we have functors

$$
\begin{gathered}
F^{s *}(\ldots) \stackrel{\text { def }}{=} R^{(s)} \otimes_{R} \_: \quad R-\bmod \longrightarrow D_{R}^{(s)}-\bmod \\
T^{s}\left(\_\right) \stackrel{\text { def }}{=} \operatorname{Hom}_{R}^{\mathrm{r}}\left(R^{(s)}, R\right) \otimes_{D_{R}^{(s)}} \_: \quad D_{R}^{(s)}-\bmod \longrightarrow R-\bmod
\end{gathered}
$$

the first of which is called the ( $s$-fold) Frobenius functor on $R$-modules.

Proposition 2.1 (Frobenius Descent). If $R$ is regular and F-finite, the functors $F^{s *}\left({ }_{-}\right)$and $T^{s}\left(_{(}\right)$are inverses of each other. Consequently they induce an equivalence between the category of $R$-modules and the category of $D_{R}^{(s)}$ modules.

Proof. To show that $F^{s *}(\ldots)$ and $T^{s}(\ldots)$ are inverses of each other it is enough to show that the natural map

$$
\Phi: R^{(s)} \otimes_{R} \operatorname{Hom}_{R}^{\mathrm{r}}\left(R^{(s)}, R\right) \longrightarrow D_{R}^{(s)}
$$

given by sending $a \otimes \varphi$ to the composition

$$
R^{(s)} \stackrel{\varphi}{\longrightarrow} R \stackrel{\tilde{a}}{\longrightarrow} R \stackrel{\mathrm{id}}{\longrightarrow} R^{(s)}
$$

(where id is the identity map on the underlying abelian group of $R$ and $R^{(s)}$ ) and the natural map

$$
\Psi: \operatorname{Hom}_{R}^{\mathrm{r}}\left(R^{(s)}, R\right) \otimes_{D_{R}^{(s)}} R^{(s)} \longrightarrow R
$$

given by sending $\varphi \otimes a$ to $\varphi(a)$ are both ring isomorphisms.

They are isomorphisms if and only if they are isomorphisms locally. Since $R$ is regular, $R^{(s)}$ is a locally free right $R$-module of finite rank [14]. Once an $R$-basis of $R^{(s)}$ is fixed, we may view $R^{(s)}$ as the set of coordinate rows of the elements of $R^{(s)}$ with respect to this basis and $\operatorname{Hom}^{\mathrm{r}}\left(R^{(s)}, R\right)$ as the set of the coordinate columns of the elements of $\operatorname{Hom}^{\mathrm{r}}\left(R^{(s)}, R\right)$ with respect to the dual basis, and $D_{R}^{(s)}$ is just the matrix algebra over $R$, so we are done by Morita duality between $R$ and $\mathrm{Mat}_{n \times n}$, as described above.

Remark 2.2. For a more explicit description of $T^{s}$ let $J_{s}$ be the left ideal of $D_{R}^{(s)}$ consisting of all $\delta$ such that $\delta(1)=0$. Then it is shown in [5, Prop. 3.12] that $T^{s}(M) \cong \operatorname{Ann}_{M} J_{s} \subseteq M$. 
Proposition 2.1 implies that the categories of $D_{R}^{(s)}$-modules for all $s$ are equivalent since each single one of them is equivalent to $R$-mod. The functor giving the equivalence between $D_{R}^{(t)}-\bmod$ and $D_{R}^{(t+s)}-\bmod$ is, of course, $F^{s *}$. Concretely, to understand the $D_{R}^{(t+s)}$-module structure on $F^{s *} M$ for some $D_{R}^{(t)}$ module $M$, we write $M \cong F^{t *} N$ for $N=T^{t}(M)$. Then $F^{s *} M=F^{(t+s) *} N=$ $R^{(t+s)} \otimes_{R} N$ carries obviously a $D_{R}^{(t+s)}$-module structure with $\delta \in D_{R}^{(t+s)}$ acting via $\delta \otimes \operatorname{id}_{N}$.

Since the union $\bigcup_{s} D_{R}^{(s)}$ is just the ring of differential operators $D_{R}$ of $R$ this implies the following proposition (after the obvious compatibilities are checked, which is straightforward and carried out in [5, Chapter 3.2]):

Proposition 2.3. Let $R$ be regular and $F$-finite. Then $F^{s *}$ is an equivalence of the category of $D_{R}$-modules with itself.

2.3. Unit $\mathbf{R}[\mathbf{F}]$-modules. We denote by $R[F]$ the ring extension of $R$ generated by a variable $F$ subject to relations $F r=r^{p} F$ for all $r \in R$. Clearly, a (left) $R[F]$-module is an $R$-module $M$ with a map of additive abelian groups $F: M \longrightarrow M$ such that $F \circ \tilde{r}=\tilde{r}^{p} \circ F$ where $\tilde{r}: R \longrightarrow R$ is the multiplication by $r$. To every $R[F]$-module $M$ is associated the map of $R$-modules $\vartheta_{M}: F^{*} M=R^{(1)} \otimes_{R} M \longrightarrow M$ sending $r \otimes m$ to $r F(m)$. The $R[F]$-module $M$ is called a unit $R[F]$-module if $\vartheta_{M}$ is bijective. Unit $R[F]$-modules are called $F$-modules in [15].

A unit $R[F]$-module $(M, \vartheta)$ carries a natural structure of $\bigcup_{s} D_{R}^{(s)}$-module and hence also of $D_{R}$-module as $D_{R}$ is a subring of $\bigcup_{s} D_{R}^{(s)}=\bigcup_{s} \operatorname{End}_{R^{p^{s}}}(R)$. Namely, set

$$
\vartheta_{s}=F^{(s-1) *}\left(\vartheta_{M}^{-1}\right) \circ F^{(s-2) *}\left(\vartheta_{M}^{-1}\right) \circ \cdots \circ \vartheta_{M}^{-1}: M \longrightarrow F^{s *} M .
$$

Every $u \in U_{s}=\operatorname{End}_{R^{p^{s}}}(R)$ acts on $F^{s *} M=R^{(s)} \otimes_{R} M$ via $u \otimes_{R} \operatorname{id}_{M}$. We let $u$ act on $M$ via $\vartheta_{s}^{-1} \circ\left(u \otimes_{R} \operatorname{id}_{M}\right) \circ \vartheta_{s}$. This action is well-defined, i.e. it depends only on $u$, but not on the particular $s[15$, p. 116].

Lemma 2.4. Let $R$ be regular and $F$-finite and let $M$ be a unit $R[F]-$ module. Then $\vartheta_{M}: F^{*} M \longrightarrow M$ is a map of $D_{R}$-modules where the $D_{R}$-structure on $F^{*} M$ is due to Theorem 2.1.

Proof. We omit a straightforward verification of this and instead refer to $[5$, Chapter 3.2]

The usual $D_{R}$-module structure on $R_{f}$ is induced, as above, by the unit $R[F]$-module structure $F: R_{f} \longrightarrow R_{f}$ sending $x \in R_{f}$ to $x^{p}$ [15, Ex. 5.2c]. The $R[F]$-module $R_{f}$ is generated by $\frac{1}{f}$ because $F^{s}\left(\frac{1}{f}\right)=\frac{1}{f^{s}}$, i.e. $R_{f}$ is a finitely generated unit $R[F]$-module (finitely generated unit $R[F]$-modules are called $F$-finite modules in [15]).

Theorem 2.5. ([15, Cor. 5.8]) Let $R$ be a regular finitely generated algebra over a commutative Noetherian regular F-finite local ring $A$ of characteristic $p>0$. 
A finitely generated unit $R[F]$-module $M$ has finite length in the category of $D_{R}$-modules. In particular, $R_{f}$ with its usual $D_{R}$-module structure has finite length in the category of $D_{R}$-modules for every $f \in R$.

\section{A chain of ideals associated to an element of a regular $F$-finite ring}

In this section $R$ is a regular and $F$-finite ring of characteristic $p>0$. For a given element $f \in R$ we aim to define a descending chain of ideals $I_{s}(f)$ indexed by the positive integers.

For this let us first assume that $R$ is a free $R^{p^{s}}$-module. Let $\left\{c_{1}^{p^{s}}, c_{2}^{p^{s}}, \ldots\right\} \subset$ $R^{p^{s}}$ be the string of coordinates of $f \in R$ with respect to some $R^{p^{s}}$-basis of $R$. We define $I_{s}(f)$ to be the ideal of $R$ generated by the set $\left\{c_{1}, c_{2}, \ldots\right\}$. This definition is independent of the choice of basis because the coordinates of $f$ with respect to one basis are linear combinations with coefficients from $R^{p^{s}}$ of the coordinates of $f$ with respect to another basis, hence the corresponding ideals are the same.

Since any regular $F$-finite $\operatorname{ring} R$ is a finite locally free $R^{p}$-module [14], Spec $R$ is covered by a finite number of open affines Spec $R_{r}$ such that $R_{r}$ is a free $R_{r}^{p}$-module (and consequently $R_{r}$ is a free $R_{r}^{p^{s}}$-module for every $s$ ). Hence we define $I_{s}(f)$ in general by glueing the local ideals defined above. This is possible due to the independence of the choice of basis in the construction.

This section is devoted to the study of the ideals $I_{s}(f)$ leading to an elementary proof of our main result in the polynomial case. But these ideals are interesting by themselves and are likely to become important, for example in tight closure theory.

A further consequence of $R$ being $F$-finite, is that the ring of differential operators of $R$ is $\bigcup_{s} D_{R}^{(s)}$ where $D_{R}^{(s)}=\operatorname{End}_{R^{p^{s}}}(R)$, according to formula (2) of Section 2. One has the following relationship between the ideals $I_{s}(f)$ and differential operators.

Lemma 3.1. $D_{R}^{(s)} \cdot f=I_{s}(f)^{\left[p^{s}\right]}$ where $D_{R}^{(s)} \cdot f \stackrel{\text { def }}{=}\left\{\delta(f) \mid \delta \in D_{R}^{(s)}\right\} \subseteq R$ and $I_{s}(f)^{\left[p^{s}\right]}$ is the ideal generated by the $p^{s}$-th powers of the elements of $I_{s}(f)$, equivalently, by the $p^{s}$-th powers of a set of generators of $I_{s}(f)$.

Proof. Since $R$ is a finitely generated $R^{p^{s}}$-module, $D_{R}^{(s)}=\operatorname{End}_{R^{p^{s}}} R$ commutes with localization with respect to any multiplicatively closed subset of $R^{p^{s}}$. Hence we may assume that $R$ is a free $R^{p^{s}}$-module. In this case $f=\sum_{i} c_{i}^{p^{s}} e_{i}$ where $\left\{e_{1}, e_{2}, \ldots\right\}$ is an $R^{p^{s}}$-basis of $R$ and $\left\{c_{1}^{p^{s}}, c_{2}^{p^{s}}, \ldots\right\}$ are the coordinates of $f$ with respect to this basis. Since $\delta(f)=\sum_{i} c_{i}^{p^{s}} \delta\left(e_{i}\right) \in I_{s}(f)^{\left[p^{s}\right]}=\left(c_{1}^{p^{s}}, c_{2}^{p^{s}}, \ldots\right)$ for every $\delta \in D_{R}^{(s)}$, we see that $D_{R}^{(s)} \cdot f \subseteq I_{s}(f)^{\left[p^{s}\right]}$. Conversely, setting $\delta_{i} \in D_{R}^{(s)}$ to be the $R^{p^{s}}$-linear map that sends $e_{i}$ to 1 and $e_{j}$ to 0 for every $j \neq i$ we see that $\delta_{i}(f)=c_{i}^{p^{s}}$, i.e. every generator of $I_{s}(f)^{\left[p^{s}\right]}$ is in $D_{R}^{(s)}$.

Lemma 3.2. $I_{s}(f)=I_{s+1}\left(f^{p}\right)$. 
Proof. It is enough to prove this after localization at every maximal ideal of $R$, hence we can assume that $R$ is local in which case $R$ is a free $R^{p}$-module. Since $1 \notin \mathfrak{m} R$, where $\mathfrak{m}$ is the maximal ideal of $R^{p}$, Nakayama's lemma implies that we can take 1 to be part of a free $R^{p}$-basis of $R$, i.e. we may assume that $R$ is a free $R^{p}$-module on some basis $\left\{e_{1}, e_{2}, \ldots\right\}$ and $e_{1}=1$.

Now let $\left\{\tilde{e}_{j}\right\}$ be an $R^{p^{s}}$-basis of $R$. Then the set of all products $\left\{e_{j, i}=\tilde{e}_{j}^{p} e_{i}\right\}$ is an $R^{p^{s+1}}$-basis of $R$. Raising the equality $f=\sum_{j} c_{j}^{p^{s}} \tilde{e}_{j}$ to the $p$-th power we get $f^{p}=\sum_{j} c_{j}^{p^{s+1}} \tilde{e}_{j}^{p}$. But $\tilde{e}_{j}^{p}=\tilde{e}_{j}^{p} \cdot 1=\tilde{e}_{j}^{p} \cdot e_{1}=e_{j, 1}$, hence the $(j, i)$-th coordinate of $f^{p}$ with respect to the basis $\left\{e_{j, i}\right\}$ is $c_{j}^{p^{s+1}}$ if $i=1$ and 0 if $i \neq 1$. Hence $I_{s}(f)$ and $I_{s+1}\left(f^{p}\right)$ are generated by the same elements.

Lemma 3.3. $I_{s}(f \tilde{f}) \subseteq I_{s}(f) I_{s}(\tilde{f}) \subseteq I_{s}(f)$ for every $\tilde{f} \in R$.

Proof. As before we may assume that $R$ is a free $R^{p^{s}}$-module on some basis $\left\{e_{1}, e_{2}, \ldots\right\}$. Multiplying the equalities $f=\sum_{i} c_{i}^{p^{s}} e_{i}$ and $\tilde{f}=\sum_{i} \tilde{c}_{i}^{p^{s}} e_{i}$ we get $f \tilde{f}=\sum_{i, j} c_{i}^{p^{s}} \tilde{c}_{j}^{p^{s}} e_{i} e_{j}$. Writing $e_{i} e_{j}=\sum_{q} \bar{c}_{q}^{p^{s}} e_{q}$, substituting this into the preceding equality and collecting similar terms we see that all the coordinates of $f \tilde{f}$ with respect to the basis $\left\{e_{i}\right\}$ are linear combinations (with coefficients from $\left.R^{p^{s}}\right)$ of the products $c_{i}^{p^{s}} \tilde{c}_{j}^{p^{s}}$. This implies that $I_{s}(f \tilde{f})$ is generated by linear combinations (with coefficients from $R$ ) of the products $c_{i} c_{j}$, hence $I_{s}(f \tilde{f}) \subseteq$ $I_{s}(f) I_{s}(\tilde{f}) \subseteq I_{s}(f)$.

Lemma 3.4. $I_{s+1}\left(f^{p^{s+1}-1}\right) \subseteq I_{s}\left(f^{p^{s}-1}\right)$.

Proof. $f^{p^{s+1}-1}=f^{p^{s+1}-p} f^{p-1}$ so $I_{s+1}\left(f^{p^{s+1}-1}\right) \subseteq I_{s+1}\left(f^{p^{s+1}-p}\right)$ by Lemma 3.3. Since $f^{p^{s+1}-p}=\left(f^{p^{s}-1}\right)^{p}$, we are done by Lemma 3.2.

Proposition 3.5. The descending chain of ideals

$$
I_{1}\left(f^{p-1}\right) \supseteq I_{2}\left(f^{p^{2}-1}\right) \supseteq \cdots
$$

stabilizes at s, i.e. $I_{s}\left(f^{p^{s}-1}\right)=I_{s+1}\left(f^{p^{s+1}-1}\right)=I_{s+2}\left(f^{p^{s+2}-1}\right)=\ldots$, if and only if there is $\delta \in D_{R}^{(s+1)}$ such that $\delta\left(\frac{1}{f}\right)=\frac{1}{f^{p}}$.

Proof. Assume $I_{s}\left(f^{p^{s}-1}\right)=I_{s+1}\left(f^{p^{s+1}-1}\right)$. On the other hand Lemma $3.2 \mathrm{im}-$ plies that $I_{s}\left(f^{p^{s}-1}\right)=I_{s+1}\left(f^{p^{s+1}}-p\right)$. Hence

$$
I_{s+1}\left(f^{p^{s+1}-p}\right)=I_{s+1}\left(f^{p^{s+1}-1}\right)
$$

and consequently $I_{s+1}\left(f^{p^{s+1}-p}\right)^{\left[p^{s+1}\right]}=I_{s+1}\left(f^{p^{s+1}-1}\right)^{\left[p^{s+1}\right]}$. This implies that $D_{R}^{(s+1)} \cdot f^{p^{s+1}-p}=D_{R}^{(s+1)} \cdot f^{s^{s+1}-1}$ by Lemma 3.1. But $f^{p^{s+1}-p}=\delta^{\prime}\left(f^{p^{s+1}-p}\right)$ where $\delta^{\prime}=1 \in D_{R}^{(s+1)}$ so we have $f^{p^{s+1}-p} \in D_{R}^{(s+1)} \cdot f^{p^{s+1}-p}$. Hence

$$
f^{p^{s+1}-p} \in D_{R}^{(s+1)} \cdot f^{p^{s+1}-1}
$$


i.e. there is $\delta \in D_{R}^{(s+1)}$ such that $\delta\left(f^{p^{s+1}-1}\right)=f^{p^{s+1}-p}$. Dividing this equality by $f^{p^{s+1}}$ and considering that every $\delta \in D_{R}^{(s+1)}$ commutes with every element of $R^{p^{s+1}}$ we get $\delta\left(\frac{f^{p^{s+1}-1}}{f^{p^{s+1}}}\right)=\frac{f^{p^{s+1}}-p}{f^{p^{s+1}}}$, i.e. $\delta\left(\frac{1}{f}\right)=\frac{1}{f^{p}}$.

Conversely, assume there is $\delta \in D_{R}^{(s+1)}$ such that $\delta\left(\frac{1}{f}\right)=\frac{1}{f^{p}}$. Multiplying this equality by $f^{p^{s+1}}$ we get $\delta\left(f^{p^{s+1}-1}\right)=f^{p^{s+1}-p}$. This implies that

$$
\begin{aligned}
D_{R}^{(s+1)} \cdot f^{p^{s+1}-p} & =D_{R}^{(s+1)} \cdot\left(\delta\left(f^{p^{s+1}-1}\right)\right)= \\
& =\left(D_{R}^{(s+1)} \cdot \delta\right)\left(f^{p^{s+1}-1}\right) \subseteq D_{R}^{(s+1)} \cdot f^{p^{s+1}-1} .
\end{aligned}
$$

Hence $I_{s+1}\left(f^{p^{s+1}-p}\right)^{\left[p^{s+1}\right]} \subseteq I_{s+1}\left(f^{p^{s+1}-1}\right)^{\left[p^{s+1}\right]}$ by Lemma 3.1. As is shown in the paragraph after next, this implies $I_{s+1}\left(f^{p^{s+1}-p}\right) \subseteq I_{s+1}\left(f^{p^{s+1}-1}\right)$. Now Lemma 3.2 implies $I_{s}\left(f^{p^{s}-1}\right) \subseteq I_{s+1}\left(f^{s^{s+1}-1}\right)$ since $f^{p^{s+1}-p}=\left(f^{p^{s}-1}\right)^{p}$. This containment and Lemma 3.4 imply that $I_{s}\left(f^{p^{s}-1}\right)=I_{s+1}\left(f^{p^{s+1}-1}\right)$.

We have proven that the existence of $\delta \in D_{R}^{(s+1)}$ such that $\delta\left(\frac{1}{f}\right)=\frac{1}{f^{p}}$ is equivalent to equality $I_{s}\left(f^{p^{s}-1}\right)=I_{s+1}\left(f^{p^{s+1}-1}\right)$. It now follows that $I_{s}\left(f^{p^{s}-1}\right)=$ $I_{s+1}\left(f^{p^{s+1}-1}\right)$ is equivalent to $I_{s}\left(f^{p^{s}-1}\right)=I_{s^{\prime}}\left(f^{p^{s^{\prime}}-1}\right)$ for all $s^{\prime}>s$ because every $\delta \in D_{R}^{(s+1)}$ automatically belongs to $D_{R}^{\left(s^{\prime}\right)}$ for all $s^{\prime}>s$.

This completes the proof of the lemma modulo the fact, used in the paragraph before the preceding one, that if $\mathcal{I}$ and $\mathcal{J}$ are two ideals of $R$ such that $\mathcal{I}^{\left[p^{s+1}\right]} \subset$ $\mathcal{J}^{\left[p^{s+1}\right]}$, then $\mathcal{I} \subset \mathcal{J}$. We are now going to prove this fact. It is enough to prove this locally, hence we can assume like in the proof of Lemma 3.2 that $R$ is a free $R^{p^{s+1}}$-module and $e_{1}=1$ is one of the free generators, i.e. $R=R^{p^{s+1}} \oplus M$ where $M$ is a free $R^{p^{s+1}}$-module. Let $\varphi: R \longrightarrow R^{p^{s+1}}$ be the map sending $r \in R$ to $r^{p^{s+1}} \in R^{p^{s+1}}$. Clearly, $\mathcal{I}^{\left[p^{s+1}\right]}=\varphi(\mathcal{I}) R$, hence $\mathcal{I}^{\left[p^{s+1}\right]} \cap R^{p^{s+1}}=$ $\varphi(\mathcal{I}) R \cap R^{p^{s+1}}=(\varphi(\mathcal{I}) \oplus \varphi(\mathcal{I}) M) \cap R^{p^{s+1}}=\varphi(\mathcal{I})$, and the same holds with $\mathcal{I}$ replaced by $\mathcal{J}$. Hence upon taking the intersection with $R^{p^{s+1}}$ the containment $\mathcal{I}^{\left[p^{s+1}\right]} \subset \mathcal{J}^{\left[p^{s+1}\right]}$ implies $\varphi(\mathcal{I}) \subset \varphi(\mathcal{J})$ which implies $\mathcal{I} \subset \mathcal{J}$ since $\varphi$ is a ring isomorphism.

We note that the proof shows that the descending chain of ideals stabilizes at the first integer $s$ such that $I_{s}\left(f^{p^{s}-1}\right)=I_{s+1}\left(f^{p^{s+1}-1}\right)$.

Corollary 3.6. The chain of ideals $I_{1}\left(f^{p-1}\right) \supseteq I_{2}\left(f^{p^{2}-1}\right) \supseteq \ldots$ stabilizes if and only if $\frac{1}{f}$ generates $R_{f}$ as a $D_{R}$-module.

Proof. If $\frac{1}{f}$ generates $R_{f}$ as a $D_{R}$-module, then $\frac{1}{f^{p}} \in D_{R} \cdot \frac{1}{f}$, i.e. there is $\delta \in D_{R}$ such that $\frac{1}{f^{p}}=\delta\left(\frac{1}{f}\right)$. Since $\delta \in D_{R}^{(s+1)}$ for some $s$, the preceding proposition shows that the chain of ideals stabilizes at $s$.

Conversely, if the chain of ideals stabilizes at $s$, the preceding proposition implies that there exists $\delta \in D_{R}^{(s+1)}$ such that $\delta\left(\frac{1}{f}\right)=\frac{1}{f^{p}}$. As is proven in the course of the proof of Lemma 3.2, locally the element 1 can always be taken as one of the elements of an $R^{p}$-basis of $R$, hence $R / R^{p}$ is a finite locally free, 
hence projective, $R^{p}$-module. Thus the natural surjection $R \longrightarrow R / R^{p}$ splits, i.e. there exists an $R^{p}$-module isomorphism $R \cong R^{p} \oplus R / R^{p}$. Let $\delta^{\prime} \in D_{R}^{(s+2)}$ be defined by $\delta^{\prime}\left(x^{p} \oplus y\right)=\delta(x)^{p}$ for all $x \in R$ (i.e. $\left.x^{p} \in R^{p}\right)$ and $y \in R / R^{p} \subseteq R$. Then $\delta^{\prime}\left(\frac{1}{f^{p}}\right)=\left(\delta\left(\frac{1}{f}\right)\right)^{p}=\left(\frac{1}{f^{p}}\right)^{p}=\frac{1}{f^{p^{2}}}$, i.e. $\frac{1}{f^{p^{2}}} \in D_{R} \cdot \frac{1}{f}$. Thus we have shown for any $f$ that $\frac{1}{f^{p}} \in D_{R} \cdot \frac{1}{f}$ implies that $\frac{1}{f^{p^{2}}} \in D_{R} \cdot \frac{1}{f}$. Hence $\frac{1}{f^{p^{s}}} \in D_{R} \cdot \frac{1}{f}$ for every $s$, by induction on $s$. But the set $\left\{\frac{1}{f^{p^{s}}}\right\}$ generates $R_{f}$ as an $R$-module.

Open Question. Let $R$ be a regular $F$-finite ring of characteristic $p>0$ and let $f \in R$ be an element. Does the chain of ideals $I_{1}\left(f^{p-1}\right) \supseteq I_{2}\left(f^{p^{2}-1}\right) \supseteq \ldots$ stabilize? Equivalently, is $R_{f}$ generated by $\frac{1}{f}$ as a $D_{R}$-module?

For an arbitrary $F$-finite ring $R$ the question is open. But in the next section we show that for a large class of regular $F$-finite rings the answer is yes! First however, we give an elementary treatment for $R$ a polynomial ring.

3.1. The case of a polynomial ring. We will use multi-index notation in the case of the polynomial ring $R=k\left[x_{1}, \ldots, x_{n}\right]$, where $k$ is a field of characteristic $p>0$. A differential operator $\delta \in D_{R}$ will be written in the right normal form, i.e. $\delta=\sum a_{\alpha \beta} x^{\alpha} D_{\beta}$, where $x^{\alpha}$ will stand for the monomial $x^{\alpha}:=x_{1}^{\alpha_{1}} \cdots x_{n}^{\alpha_{n}}$, $D_{\beta}$ will denote the differential operator $D_{\beta}:=D_{\beta_{1}, 1} \cdots D_{\beta_{n}, n}$ and all but finitely many $a_{\alpha \beta} \in k$ are zero.

Theorem 3.7. Let $R=k\left[x_{1}, \ldots, x_{d}\right]$ be a polynomial ring in $x_{1}, \ldots, x_{d}$ over a perfect field $k$ of characteristic $p>0$ and let $f \in R$ be any element. The chain of ideals $I_{1}\left(f^{p-1}\right) \supseteq I_{2}\left(f^{p^{2}-1}\right) \supseteq \ldots$ stabilizes.

Proof. The monomials $\left\{x^{\alpha}=x_{1}^{\alpha_{1}} \cdots x_{d}^{\alpha_{d}} \mid 0 \leq \alpha_{i} \leq p^{s}-1\right\}$ form an $R^{p^{s}}$-basis of $R$. Let $f^{p^{s}-1}=\sum_{\alpha} c_{\alpha}^{p^{s}} x^{\alpha}$, so that $I_{s}\left(f^{p^{s}-1}\right)$ is generated by the set $\left\{c_{\alpha}\right\}$. No monomials on the right side of the equation $f^{p^{s}-1}=\sum_{\alpha} c_{\alpha}^{p^{s}} x^{\alpha}$ get cancelled as a result of reducing similar terms, hence $\operatorname{deg} f^{p^{s}-1} \geq \operatorname{deg} c_{\alpha}^{p^{s}}$ for every $\alpha$. This inequality translates to $\left(p^{s}-1\right) \operatorname{deg} f \geq p^{s} \operatorname{deg} c_{\alpha}$, i.e. $\operatorname{deg} c_{\alpha} \leq \frac{p^{s}-1}{p^{s}} \operatorname{deg} f$. Hence $\operatorname{deg} c_{\alpha}<\operatorname{deg} f$. Thus the ideals $I_{s}\left(f^{p^{s}-1}\right)$ for every $s$ are generated by polynomials of degrees less than $\operatorname{deg} f$ which is independent of $s$. The set of polynomials of degrees less than $\operatorname{deg} f$ is a finite dimensional $k$-vector space and the intersections of the ideals $I_{s}\left(f^{p^{s}-1}\right)$ with this vector space form a descending chain of subspaces which stabilizes because the space is finite dimensional. Hence $I_{1}\left(f^{p-1}\right) \supseteq I_{2}\left(f^{p^{2}-1}\right) \supseteq \ldots$ stabilizes.

Corollary 3.8. Let $R=k\left[x_{1}, \ldots, x_{d}\right]$ be a polynomial ring in $x_{1}, \ldots, x_{d}$ over an arbitrary field $k$ of characteristic $p>0$ and let $f \in R$ be any element. $R_{f}$ is generated by $\frac{1}{f}$ as a $D_{R \mid k}-$ module.

Proof. If $k$ is perfect, we are done by Corollary 3.6 and Theorem 3.7. In the general case, let $K$ be the perfect closure of $k$. Since $K$ is perfect, there is a differential operator $\delta=\sum a_{\alpha \beta} x^{\alpha} D_{\beta}$ with coefficients $a_{\alpha \beta} \in K$ such that $\delta\left(\frac{1}{f}\right)=\frac{1}{f^{p}}$. This is equivalent to the fact that a system of finitely many linear 
equations with coefficients in $k$ has solutions in $K$, where the non-zero coefficients $a_{\alpha \beta}$ of $\delta$ are thought of as the unknowns of the system. (For example, if $f=x_{1}$, we may be looking for a solution in the form $\delta=a D_{p-1,1}$, so we get an equation $\delta\left(\frac{1}{x}\right)=\frac{1}{x^{p}}$. Since $\delta\left(\frac{1}{x_{1}}\right)=a \frac{1}{x_{1}^{p}}$, the corresponding linear system is just one equation $a=1$.) The system has a solution in $K$, namely, the coefficients of $\delta$. Hence it is consistent, so it must have a solution in $k$ because the coefficients of the linear system are in $k$ (they depend only on the coefficients of $f$ ). So there is a differential operator $\delta^{\prime}$ with coefficients in $k$ such that $\delta^{\prime}\left(\frac{1}{f}\right)=\frac{1}{f^{p}}$.

We conclude this section with an example. Let $R=k\left[x_{1}, x_{2}, x_{3}, x_{4}\right]$ where $k$ is a field of characteristic $p>0$ and let $f=x_{1}^{2}+x_{2}^{2}+x_{3}^{2}+x_{4}^{2}$. In characteristic 0 , as is pointed out in the Introduction, $\frac{1}{f^{2}}$ does not belong to the $D_{R^{-} \text {-submodule }}$ of $R_{f}$ generated by $\frac{1}{f}$. But in characteristic $p>0$ we are going to find a differential operator $\delta \in D_{R}$ such that $\delta\left(\frac{1}{f}\right)=\frac{1}{f^{p}}$ just by investigating the monomials appearing in $f^{p-1}$.

- If 4 divides $p-1$, then $f^{p-1}$ contains the term $a_{\alpha} x^{\alpha}$ where

$$
a_{\alpha}=\frac{(p-1) !}{\left(\frac{p-1}{4} !\right)^{4}} \neq 0 \text { and } \alpha=\left(\frac{p-1}{2}, \frac{p-1}{2}, \frac{p-1}{2}, \frac{p-1}{2}\right) .
$$

- If 4 does not divide $p-1$, then $f^{p-1}$ contains the term $a_{\alpha} x^{\alpha}$ where

$$
a_{\alpha}=\frac{(p-1) !}{\left(\frac{p+1}{4} !\right)^{2}\left(\frac{p-3}{4} !\right)^{2}} \neq 0 \text { and } \alpha=\left(\frac{p+1}{2}, \frac{p+1}{2}, \frac{p-3}{2}, \frac{p-3}{2}\right) .
$$

Notice that $\frac{1}{a_{\alpha}} D_{\alpha}\left(f^{p-1}\right)=1$ because all other monomials appearing in $f^{p-1}$ contain some $x_{i}$ raised to a power smaller than the power of $\frac{\partial_{i}}{\partial x_{i}}$ in $D_{\alpha}$ hence $D_{\alpha}$ annihilates all other monomials. The differential operator $\delta=\frac{1}{a_{\alpha}} D_{\alpha}$ commutes with $f^{p}$ so, dividing the equation $\delta\left(f^{p-1}\right)=1$ by $f^{p}$ we get the desired result.

\section{The case of a regular finitely generated algebra over an $F$-finite regular local ring}

Here we prove the central result of our paper using the techniques surveyed in Section 2.

Theorem 4.1. Let $R$ be a regular finitely generated algebra over an $F$-finite regular local ring of characteristic $p>0$. Let $f \in R$ be a nonzero element. Then the $D_{R}$-module $R_{f}$ is generated by $\frac{1}{f}$.

Proof. For any $D_{R}$-submodule $M \subseteq R_{f}$ we identify $F^{*} M$ with its isomorphic image in $R_{f}$ via the natural $D_{R}$-module isomorphism $\vartheta: F^{*} R_{f} \longrightarrow R_{f}$ of Lemma 2.4 (with $R_{f}$ viewed as a unit $R[F]$-module given by the map $F: R_{f} \longrightarrow R_{f}$ that sends every $x \in R_{f}$ to $x^{p}$ ). Then $F^{*} M$ is $R$-generated by the elements $m^{p}$ for $m \in M \subseteq R_{f}$. By Frobenius descent (Proposition 2.1), $F^{*} M$ is a $D_{R^{-}}$submodule of $R_{f}$. 
Let $M=D_{R} \cdot \frac{1}{f}$. We claim that $M \subseteq F^{*} M$. Because $F^{*} M$ is a $D_{R^{-}}$ submodule of $R_{f}$, it is enough to show that $\frac{1}{f} \in F^{*} M$. But $\frac{1}{f} \in M$ implies $\left(\frac{1}{f}\right)^{p}=\frac{1}{f^{p}} \in F^{*} M$, hence $f^{p-1} \cdot \frac{1}{f^{p}}=\frac{1}{f} \in F^{*} M$. This proves the claim.

Now we get an ascending chain of $D_{R}$-submodules of $R_{f}$ :

$$
M \subseteq F^{*} M \subseteq F^{2 *} M \subseteq F^{3 *} M \subseteq \cdots
$$

The fact that $\frac{1}{f} \in M$ implies $\frac{1}{f^{p^{s}}}=F^{s}\left(\frac{1}{f}\right) \in F^{s *} M$, hence the union of the chain must be all of $R_{f}$. Thus it is enough to show that $M=F^{*} M$ since then $M=F^{s *} M$ for all $s$, hence $M=R_{f}$ as claimed. Assume otherwise, that is assume that the inclusion $M \subsetneq F^{*} M$ is strict. Then all the inclusions of (3) must be strict since $F^{* *}\left(\_\right)=R^{(s)} \otimes_{R}\left({ }_{-}\right)$and $R^{(s)}$ is a faithfully flat right $R$-module. But this contradicts the fact that by Theorem 2.5 the length of $R_{f}$ as a $D_{R}$-module is finite.

Corollary 4.2. Let $R$ be a regular finitely generated algebra over an $F$-finite regular local ring of characteristic $p>0$. Let $f \in R$ be any element. The descending chain of ideals $I_{1}\left(f^{p-1}\right) \supseteq I_{2}\left(f^{p^{2}-1}\right) \supseteq \ldots$ defined in the preceding section stabilizes.

Proof. This follows from Corollary 3.6.

Theorem 4.1 also follows from the following more general observation which was inspired by [9, Prop. 15.3.4], which in the notation of Theorem 4.3 states that if $F^{*} M \subseteq M$ then $M$ is also a unit $R[F]$-submodule.

Theorem 4.3. Let $R$ be a regular finitely generated algebra over an $F$-finite regular local ring of characteristic $p>0$. Let $N$ be a finitely generated unit $R[F]-$ module. Suppose $M \subseteq N$ is a $D_{R}$-submodule such that $M \subseteq F^{*} M$ (we identify $F^{*} M \subseteq F^{*} N$ with its image in $N$ via the structural isomorphism $\vartheta: F^{*} N \longrightarrow N$ of $N)$. Then $M$ is a unit $R[F]$-submodule.

Proof. $M$ being a unit $R[F]$-submodule of $N$ just means that the inclusion $M \subseteq F^{*} M$ is in fact an equality. If the inclusion is strict, then all the inclusions $F^{s *} M \subsetneq F^{(s+1) *} M$ are strict as well because they are obtained by tensoring $M \subseteq$ $F^{*} M$ with the faithfully flat $R$-module $R^{(s)}$. The resulting strictly increasing infinite chain

$$
M \subsetneq F^{*} M \subsetneq F^{2 *} M \subsetneq F^{3 *} M \subsetneq \cdots
$$

contradicts the finiteness of the length of $N$ as a $D_{R}$-module.

To obtain Theorem 4.1 from this just note that $M=D_{R} \cdot \frac{1}{f}$ satisfies $M \subseteq F^{*} M$ and contains the $R[F]$-module generator $\frac{1}{f}$ of $R_{f}$.

An $R$-submodule $N_{0}$ of a unit $R[F]$-module $N$ is called a root, if $N_{0}$ is finitely generated as an $R$-module, $N_{0} \subseteq F^{*} N_{0}$ and $\bigcup_{s} F^{s *} N_{0}=N$. The existence of a root is equivalent to $N$ being finitely generated as a unit $R[F]-$ module [5, Cor. $2.12]$. 
Corollary 4.4. With the same assumptions as in Theorem 4.3, if $n_{1}, \ldots, n_{t}$ are generators of a root of a finitely generated unit $R[F]$-module $N$, then $n_{1}, \ldots, n_{t}$ generate $N$ as a $D_{R}$-module.

Proof. By Theorem 4.3 it is enough to check that the $D_{R}$-submodule $M \stackrel{\text { def }}{=}$ $D_{R} \cdot\left\langle n_{1}, \ldots, n_{t}\right\rangle$ satisfies $M \subseteq F^{*} M$ and contains the $R[F]$-module generators $n_{1}, \ldots, n_{t}$ of $N$. The second statement is trivial and for the first one observes that, by definition of root, one can write $n_{i}=\sum r_{j} F\left(n_{j}\right)$ for some $r_{j} \in R$. Noting that $F\left(n_{j}\right) \in F^{*} M$ we conclude $n_{i} \in F^{*} M$ for all $i$ as required.

The above corollary is a generalization of Theorem 4.1 in that $R_{f}$ is a finitely generated $R[F]$-module with root generated by $n=\frac{1}{f}$.

\section{The case of a finitely generated algebra over a formal power series ring}

The purpose of this section is to prove that $R_{f}$ is $D_{R \mid k}$-generated by $\frac{1}{f}$ in an important case that is not covered by our previous results. Namely for $R$ a finitely generated algebra over a power series ring $A=k\left[\left[x_{1}, \ldots, x_{n}\right]\right]$ over a field $k$ of positive characteristic. The improvement is that we no longer assume that $k$ is perfect.

Fixing the notation just introduced we further denote by $k\left[\left[A^{p^{s}}\right]\right]=$ $k\left[\left[x_{1}^{p^{s}}, \ldots, x_{n}^{p^{s}}\right]\right]$ the $k$-subalgebra of $A$ consisting of all the power series in $x_{1}^{p^{s}}, \ldots, x_{n}^{p^{s}}$ with coefficients in $k$. By $k\left[\left[A^{p^{s}}\right]\right]\left[R^{p^{s}}\right]$ we denote the $k\left[\left[A^{p^{s}}\right]\right]-$ subalgebra of $R$ generated by the $p^{s}$-th powers of all the elements of $R$. The fact that $A$ is a finite $k\left[\left[A^{p^{s}}\right]\right]$-module implies that $R$ is a finite $k\left[\left[A^{p^{s}}\right]\right]\left[R^{p^{s}}\right]-$ module. Hence the ring of the $k\left[\left[A^{p^{s}}\right]\right]\left[R^{p^{s}}\right]$-linear differential operators of $R$ is just $\operatorname{End}_{k\left[\left[A^{p^{s}}\right]\right]\left[R^{p^{s}}\right]}(R)$ due to formula (1) of Section 2. Every $k\left[\left[A^{p^{s}}\right]\right]\left[R^{p^{t}}\right]$-linear differential operator of $R$ is automatically $k$-linear so $D_{R \mid k} \supseteq V(R, k)$ where

$$
V(R, k)=\bigcup_{s} \operatorname{End}_{k\left[\left[A^{p^{s}}\right]\right]\left[R^{p^{s}}\right]}(R)
$$

As is pointed out in [15, Ex. 5.3c], we do not know whether this containment is always an equality (but it is if $R=A$ ).

Let $k^{*}=k^{1 / p^{\infty}}$ be the perfect closure of $k$, let $A^{*}=k^{*}\left[\left[x_{1}, \ldots, x_{n}\right]\right]$ and let $R^{*}=A^{*} \otimes_{A} R$ where $A^{*}$ is regarded as an $A$-algebra via the natural inclusion $k\left[\left[x_{1}, \ldots, x_{n}\right]\right] \subseteq k^{*}\left[\left[x_{1}, \ldots, x_{n}\right]\right]$. Since $R$ is a finitely generated $A$-algebra, $R^{*}$ is a finitely generated, hence Noetherian, $A^{*}$-algebra.

Theorem 5.1. With notation as above, let $R$ be a finitely generated A-algebra such that $R^{*}$ is regular. Then $R_{f}$ is generated by $\frac{1}{f}$ as a $D_{R \mid k}$-module.

Proof. Since $V(R, k)$ is a subring of $D_{R \mid k}$, it is enough to prove that $\frac{1}{f}$ generates $R_{f}$ as a $V(R, k)$-module. According to [15, p. 129],

$$
D_{R^{*}}=R^{*} \otimes_{R} V(R, k)
$$


and there is a functor

$$
V(R, k)-\bmod \stackrel{N \mapsto R^{*} \otimes_{R}}{\longrightarrow} D_{R^{*}-\bmod }
$$

where for each $V(R, k)$-module $N$ the $D_{R^{*}}$-module structure on $R^{*} \otimes_{R} N$ is defined as follows: if $\delta \in D_{R^{*}}, r \otimes n \in R^{*} \otimes_{R} N$ and $\delta(r)=\sum_{i}\left(r_{i} \otimes v_{i}\right)$, where $r_{i} \in R^{*}, v_{i} \in V(R, k)$, then $\delta(r \otimes n)=\sum_{i}\left(r_{i} \otimes v_{i}(n)\right)$.

Since $A^{*}$ is flat over $A$ [16, Thm. $\left.22.3(\beta)(1)\left(3^{\prime}\right)\right]$ and local, it is faithfully flat over $A$, hence $R^{*}$ is faithfully flat over $R$. Let $M \subset R_{f}$ be the $V(R, k)$ submodule generated by $\frac{1}{f}$. Since $R_{f}^{*}=R^{*} \otimes_{R} R_{f}$, we conclude that $R^{*} \otimes_{R} M$ is

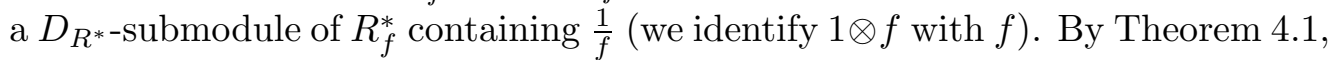
$R^{*} \otimes_{R} M=R_{f}^{*}$. Since $R_{f}^{*}$ is faithfully flat over $R$, we conclude that $M=R_{f}$.

Theorem 5.2. With notation as above, let $R$ be a finitely generated A-algebra such that $R^{*}$ is regular. If $n_{1}, \ldots, n_{t}$ are generators of a root of a finitely generated unit $R[F]-$ module $N$, then $n_{1}, \ldots, n_{t}$ generate $N$ as a $D_{R \mid k}$-module.

Proof. It is enough to prove that $n_{1}, \ldots, n_{t}$ generate $N$ as a $V(R, k)$-module. If not, let $M \subseteq N$ be the $V(R, k)$-submodule of $N$ generated by $n_{1}, \ldots, n_{t}$. Since $R^{*}$ is faithfully flat over $R$, we conclude that $R^{*} \otimes_{R} M$ is a $D_{R^{*} \text {-submodule of }}$ $R^{*} \otimes_{R} N$ different from $R^{*} \otimes_{R} N$. But this contradicts Corollary 4.4 since $R^{*} \otimes_{R} N$ contains $1 \otimes n_{1}, \ldots, 1 \otimes n_{t}$ and these elements generate a root of $R^{*} \otimes_{R} N$.

The following special case of Theorems 5.1 and 5.2 deserves to be stated separately.

Corollary 5.3. Let $R$ be a finitely generated algebra over a field $k$ of characteristic $p>0$ such that $k^{1 / p^{\infty}} \otimes_{k} R$ is regular. Then

(a) $R_{f}$, for any $f \in R$, is generated by $\frac{1}{f}$ as a $D_{R \mid k}$-module.

(b) More generally, if $n_{1}, \ldots, n_{t}$ are generators of a root of a finitely generated unit $R[F]$-module $N$, then $n_{1}, \ldots, n_{t}$ generate $N$ as a $D_{R \mid k}-$ module.

\section{References}

[1] J. Alvarez-Montaner and G. Lyubeznik, A surprising fact about D-modules in characteristic $p>0$, arXiv:math.AC/0407464.

[2] I. N. Bernšte $\breve{i n}$, Analytic continuation of generalized functions with respect to a parameter, Funkcional. Anal. i Priložen., 6, no. 4, (1972) 26-40.

[3] P. Berthelot, D-modules arithmétiques. II. Descente par Frobenius, Mém. Soc. Math. Fr. (N.S.) (2000), no. 81, vi+136.

[4] J. E. Björk, Rings of differential operators, North Holland Mathematics Library, 21. North-Holland Publishing Co., Amsterdam-New York, 1979.

[5] M. Blickle, The intersection homology D-module in finite characteristic, Ph.D. thesis, University of Michigan, 2001, arXiv:math.AG/0110244.

[6] - The intersection homology D-module in finite characteristic, Math. Ann. 328 (2004), 425-450.

[7] — D-module generation in positive characteristic via Frobenius descent, arXiv:math.AC/0408124.

[8] R. Bøgvad, Some results on D-modules on Borel varieties in characteristic $p>0$, J. Algebra 173 (1995), no. 3, 638-667. 
[9] M. Emerton and M. Kisin, Riemann-Hilbert correspondence for unit $\mathcal{F}$-crystals, Astérisque, 293 (2004).

[10] A. Grothendieck and J. Dieudonné, Éléments de géométrie algébrique IV. Étude locale des schémas et des morphismes de schémas, (French) Inst. Hautes tudes Sci. Publ. Math. 32 (1967).

[11] B. Haastert, Über Differentialoperatoren und D-Moduln in, positiver Charakteristik, Manuscripta Math. 58 (1987), no. 4, 385-415.

[12] - On direct and inverse images of D-modules in prime characteristic, Manuscripta Math. 62 (1988), no. 3, 341-354.

[13] N. M. Katz, Nilpotent connections and the monodromy theorem: Applications of a result of Turrittin, Publ. Math., Inst. Hautes Etud. Sci. 39 (1970), 175-232.

[14] E. Kunz, Characterization of regular local rings in characteristic p, Amer J. Math. 91 (1969), 772-784.

[15] G. Lyubeznik, $\mathcal{F}$-modules: an application to local cohomology and D-modules in characteristic $p>0$, J. Reine Angew. Math. 491 (1997), 65-130.

[16] H. Matsumura, Commutative Ring Theory, Cambridge Studies in Advanced Mathematics, 8, Cambridge University Press, 1992.

[17] S. P. Smith, Differential operators on the affine and projective lines in characteristic $p>0$, Séminaire d'algèbre Paul Dubreil et Marie-Paule Malliavin, 37ème année (Paris, 1985), Springer, Berlin, 1986, pp. 157-177.

[18] _ The global homological dimension of the ring of differential operators on a nonsingular variety over a field of positive characteristic, J. Algebra 107 (1987), no. 1, 98-105.

[19] U. Walther, Bernstein-Sato polynomials versus cohomology of the Milnor fiber for generic hyperplane arrangements, Compositio Math. 141 (2005), no. 1, 121-145.

[20] T. Yano, On the theory of b-functions, Publ. Res. Inst. Math. Sci. 14 (1978), no. 1, 111-202.

[21] A. Yekutieli, An explicit construction of the Grothendieck residue complex, appendix by Pramathanath Sastry. Astérisque No. 208 (1992).

Departament de Matemàtica Aplicada I, Universitat Politècnica de Catalunya, Avinguda Diagonal 647, Barcelona 08028, Spain

E-mail address: Josep.Alvarez@upc.es

FB6 Mathematik, Universität Duisburg-Essen, 45117 Essen, Germany

E-mail address: manuel.blickle@uni-essen.de

Department of Mathematics, University of Minnesota, Minneapolis, MN 55455

E-mail address: gennady@math.umn.edu 